\title{
Penetration Test for the 3G Internet Service Provider and Free Basics Service by Facebook
}

\author{
https://doi.org/10.3991/ijim.v15i03.18905 \\ Salim M. Zaki \\ Dijlah University College, Baghdad, Iraq \\ salimzki@gmail.com
}

\begin{abstract}
The number of devices connected to the Internet via mobile devices is increasing every day. The cost of mobile data over $3 \mathrm{G}$ and $4 \mathrm{G}$ networks is high in some countries, which pushes many users to browse the internet through text-only service. Facebook proposed a free basics service that allows users to browse Facebook for free but without showing images. This research formats the images on web pages using a method that transfers images over text-only networks. This will allow users with low income or slow connections to surf text service over the internet with images supported. The main objective is to test the free basics service by Facebook and Internet Service Provider over $3 \mathrm{G}$ networks, additionally proposing an image format for text-only networks. The proposed algorithm converts .png and .jpg images and posts them on the Facebook page. The results from the Facebook page show images that can be seen over $3 \mathrm{G}$ networks with the free mode enabled.
\end{abstract}

Keywords - Facebook, penetration test, mobile data, social network security

\section{Introduction}

Social networks are becoming an essential part of people's everyday life [1]. Social networks' platforms increase with the number of users and offer different services to those users. However, this comes at a cost to privacy and security, and, therefore, securing mobile applications and social networks is vitally important in the development process [2].

Facebook founded the Internet.org organization [3] with Free Basics service in collaboration with cell phone service suppliers in several developing nations [4] [5]. The concept of the Digital Divide [6] and Internet.org sets a goal for customers of 3G or $4 \mathrm{G}$ networks to be able to access many web benefits on their cell phone programs without subscribing to paid internet service. The service has expanded to more than 60 countries across Asia, Africa, and South and Central America, with 25 new countries included last year. Facebook claims that its objective with Free Basics is to connect more individuals to the internet, with an end goal to control the computerized gap between people and web services [7] [8] [3]. Images are an attractive element of any web page, which leads to the desire to find a way to allow people to browse images on a text-only service as users demand images with good or high resolution [9]. 
The motivation behind this study is to check the ability of text-only services to accept special images and continue showing those images to users. Privacy on social media is a vital topic nowadays due to large amounts of private data exchanged over social media platforms such as Facebook [10] [11]. This study aims to test the quality of Free Basics service as few research studies have tested the quality and the ability to bypass the limitations of text-only service. Although there are research studies focusing on Free Basics Service by Facebook such as [12] [13] [14] [15], to the best of our knowledge, there has been no research on a penetration test for free basics services. Additionally, this research is in the scope of "bug bounty," which is a program that rewards experts for finding software bugs in websites, apps and services [16][17]. Therefore, this study provides a technique for posting images to Facebook and downloading them as .png files over free modes on $3 \mathrm{G}$ networks.

The major contributions of the paper are summarized as follows:

1. Developing an algorithm that provides images for text-only networks.

2. Images can be seen on Facebook pages even when enabled via a free mode.

3. Testing the free basics service provided by Facebook and Internet Service provider (ISP) over $3 \mathrm{G}$.

\section{Background}

\subsection{What is the service architecture of free basics?}

As shown in Fig. 1, the Free Basics administration includes three autonomous service suppliers: (I) the cellular company that agrees to convey information for any Free Basics administration at no expense to the end client. (II) Proxy servers by Facebook to manage free basics services. (III) the service provided by participants in the service such as Wikipedia and Facebook social media site.

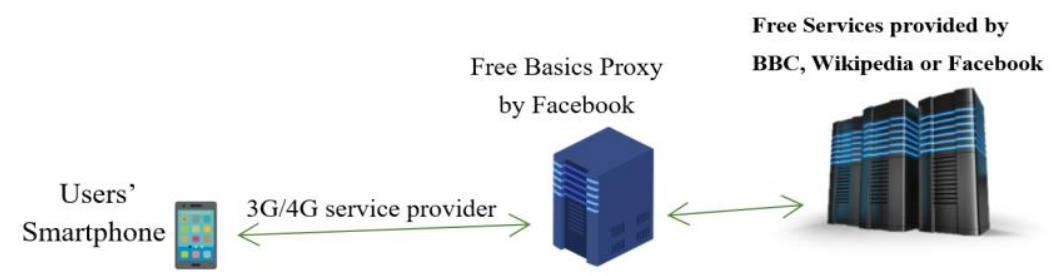

Fig. 1. Architecture of Free Basics Service by Facebook 


\subsection{Image file format}

Files provide the vital mechanism for storing, archiving and exchanging image data. And the choice of the correct file format is an important decision. Today, there exists a wide range of standardized file formats, and developers can always find at least one image format suitable for their applications [18] [19]. The selection of file format is not an easy choice, but there are criteria in place to choose the correct format for the application:

- Type of Image File: Includes binary image, color image, greyscale and special images.

- Size on Disk and Compression Technique: One important factor is maximum size of the image. The storage requirement of an image file plays an important role in selecting the appropriate image file format for an application. The compression technique and lossy or lossless is important.

- Compatibility and Domain: Image file format compatibility is vital when used in global application of place such as internet or archive. The file format should achieve this factor by choosing the best format that is compatible in different domains such as web browsers and file viewing and editing software.

Table 1 offers a comparison of the most commonly used image file formats on the internet, mobile devices and personal computers. Tagged Image File Format is suitable for archiving but not supported on the web; on the other hand, Graphics Interchange Format is supported on the web, but with low resolution. The suitable solution is Portable Network Graphics with good resolution and a size that is suitable for web design. The Joint Photographic Experts Group became very popular among users and designers due to its good features such as resolution and size [20] [19].

Table 1. Summary of Image File Format Features

\begin{tabular}{|c|c|c|c|c|}
\hline Image Format & Extension & Good Features & Shortcomings & Application \\
\hline $\begin{array}{l}\text { Tagged Image } \\
\text { File Format }\end{array}$ & tif, tiff & $\begin{array}{ll}\text { - } & \text { Storing raster graphics imag- } \\
\text { es. } \\
\text { Widely used for different } \\
\text { applications. }\end{array}$ & $\begin{array}{l}\text { No wide support } \\
\text { in web browsers. }\end{array}$ & $\begin{array}{l}\text { Scanning, word } \\
\text { processing, optical } \\
\text { character recogni- } \\
\text { tion. }\end{array}$ \\
\hline $\begin{array}{l}\text { Graphics Inter- } \\
\text { change Format }\end{array}$ & gif & $\begin{array}{ll}\text { - } & \text { Wide support on web. } \\
\text { - } & \text { Static and dynamic. } \\
\text { Portability on many operating } \\
\text { systems. }\end{array}$ & $\begin{array}{l}\text { Low resolution } \\
\text { and poor colors. }\end{array}$ & $\begin{array}{l}\text { Widely used on web } \\
\text { and operating sys- } \\
\text { tems. }\end{array}$ \\
\hline $\begin{array}{l}\text { Portable Network } \\
\text { Graphics }\end{array}$ & png & $\begin{array}{l}\text { - } \quad \text { Widely used on Internet. } \\
\text { - } \\
\text { Multi-color support. } \\
\text { Alpha channel for transparen- } \\
\text { cy. }\end{array}$ & $\begin{array}{l}\text { No dynamic image } \\
\text { in one file as is the } \\
\text { case in GIF for- } \\
\text { mat. }\end{array}$ & Web design. \\
\hline $\begin{array}{l}\text { Joint Photo- } \\
\text { graphic Experts } \\
\text { Group }\end{array}$ & jpg, jpeg & $\begin{array}{l}\text { Most widely used image } \\
\text { format. } \\
\text { - Good resolution and colors. }\end{array}$ & $\begin{array}{l}\text { Not well suited for } \\
\text { line drawings and } \\
\text { other textual or } \\
\text { iconic graphics. }\end{array}$ & $\begin{array}{l}\text { Digital photog- } \\
\text { raphy, Internet and } \\
\text { social media. }\end{array}$ \\
\hline
\end{tabular}




\subsection{Data URIs and inline images}

For images and SVG files, the number of HTTP requests can be decreased instead of downloading a separate file of the image file; the image data can be represented in a format that can be included inside HTML5 or CSS code [21][22].

Encoding image data in a string format is done with Base64 encoding. Base64 is a collection of comparable binary-to-text encoding structures that represent binary data in an American Standard Code for Information Interchange (ASCII) string format by translating it into a radix-64 format. Base 64 encoding schemes are used to encode binary data to be stored and transferred over media that are designed to deal with ASCII. This is to guarantee that the data is intact without amendment during the transmission process. A number of applications use Base64 such as email using MIME, and storing composite data in XML [22][23].

The type of data to be encoded is specified first in format of Data URI, such as image/png; the base64 means base-64 encoding. If the base64 is lost, standard URL encoding is used instead. An example of Base64 encoding in an image tag of HTML:

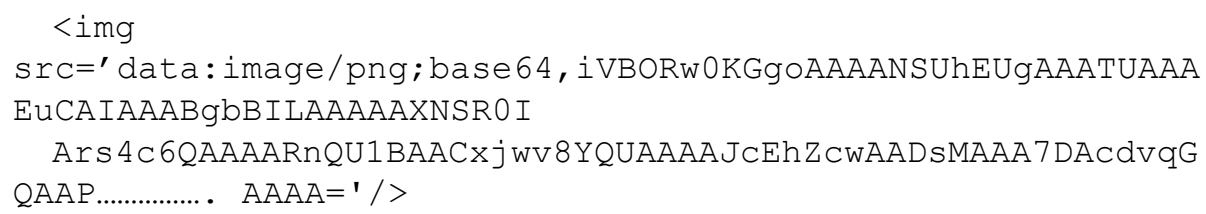

\section{$3 \quad$ Methodology}

The software takes an image as input and determines the image's dimensions. The second step is to read the image pixel by pixel to determine the location of each pixel and color value in each pixel. Then, the software rewrites the pixel values in textual representation in the same locations as the original pixels but inside HTML5 canvas element. Fig. 2 shows those steps in the algorithm. 


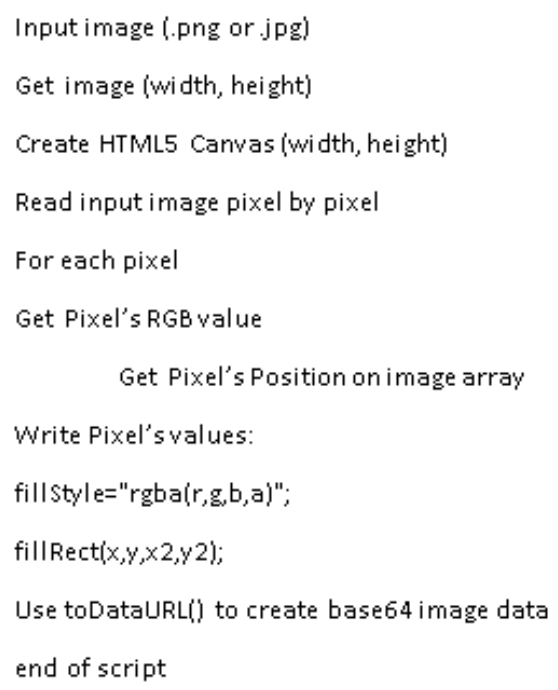

Fig. 2. Algorithm of Transferring .png and .jpg formats to Images for Free Basics Service

Fig. 3 depicts the output image described as HTML5 Canvas and JavaScript code describing RGB values of each pixel inside an HTML5 document. There is no need for an attached image file represented by its path on a server or a link on the internet to imbed an image inside a webpage. The produced JavaScript code will describe the image for the browser that translates it to a drawing image inside the HTML5 canvas element.

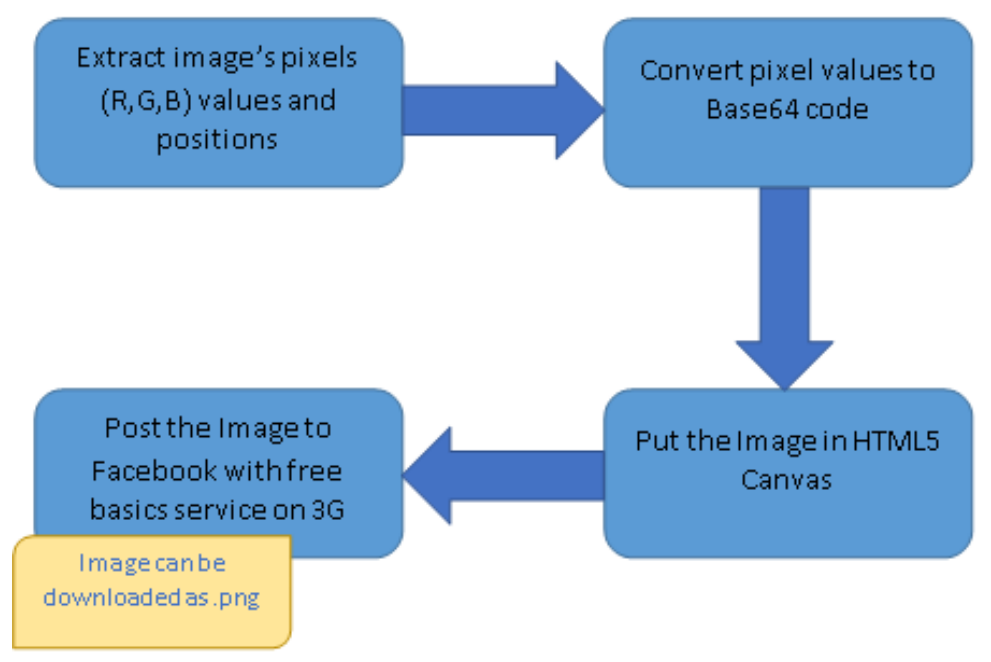

Fig. 3. Main Steps of Proposed Algorithm 


\section{$4 \quad$ Testing and Results}

The testing is done in two different ways:

The first method involves changing settings in the most popular browsers, Firefox Mozilla and Google Chrome, to block images from being viewed in the browser. Normal images used on webpages with extensions of .png, gif and .jpg can be blocked easily using browser settings or add-ons for controlling webpages. Meanwhile, the proposed representation of the images could not be blocked and continued showing on the webpage.

The second method involves testing the free basics service from Facebook to show that the image representation proposed in this research can be shown on smartphones by enabling a free mode that shows text only, and no images or videos can be seen on $3 \mathrm{G}$ networks.

\subsection{Test on web browsers}

The first section of results is taken from examining produced images inside browsers by applying settings of browsers and installed add-ons of web development. These results show the ability of some images to avoid image-blocking settings.

In the beginning, the proposed algorithm is used to represent the input image pixel by pixel inside the canvas and then convert it to Base64 image. Fig. 4 below shows the button, when clicked, converts the image above the button to the Base64 image below the button.

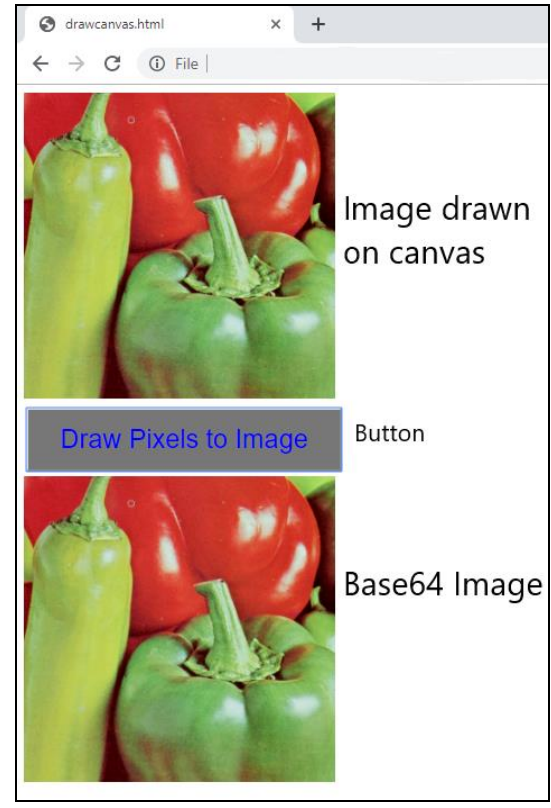

Fig. 4. Drawing Image Pixels 
The converted image has a similar resolution and is similar in size both in canvas and output. Both images can be saved with a .png extension. This is useful for later usage to be posted on the Facebook page created for testing, as will be discussed later in this section. The representation of each pixel's location and RGB values requires many lines of codes. This increases the size of the HTML document, but with the advantage of showing the image, but as a canvas. Even though, this large size of representation in canvas is a transition phase to produce the required image.

Fig. 5 shows the inspection of converter inside the browser where values of drawn pixels of an image are represented inside a canvas. The Base64 representation is used inside $s r c$ attribute of $\langle i m g\rangle$ tag.
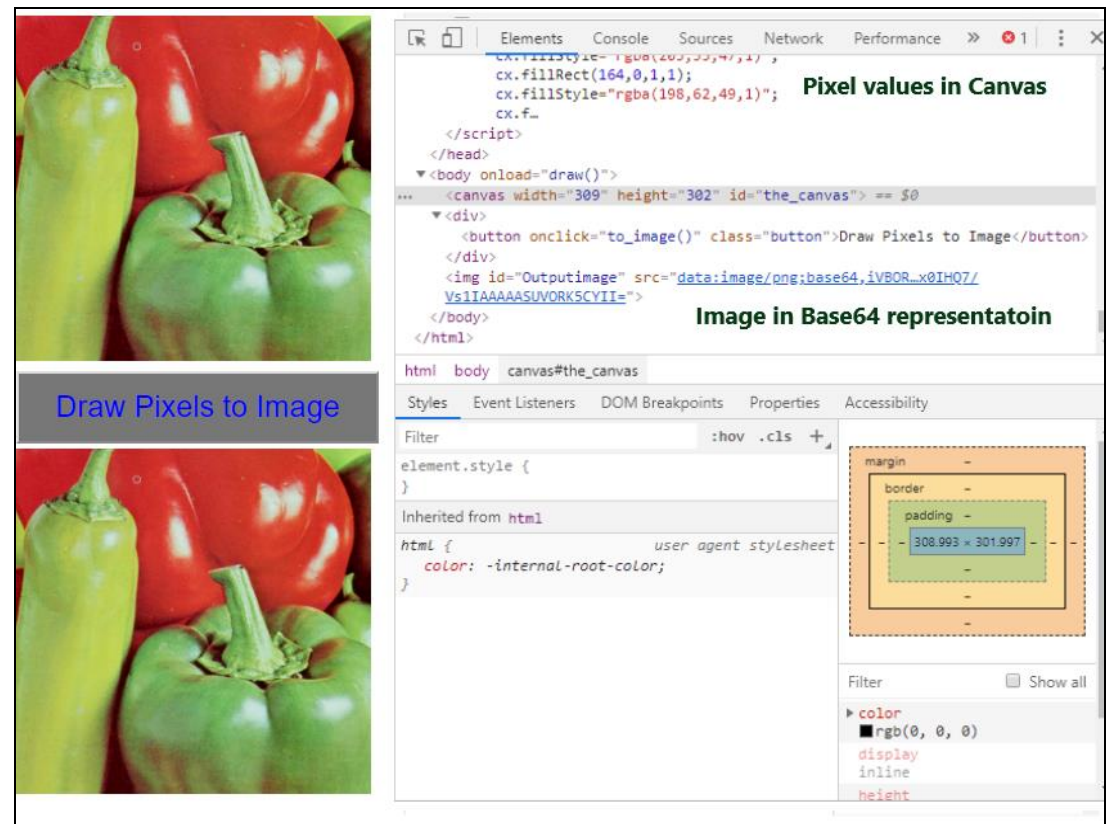

Fig. 5. Inspecting Image Conversion Details in Browser

Fig. 6 shows hiding and disabling images using an add-on installed on Google Chrome to manage web development issues. The image is drawn pixel by pixel and represented in a canvas, never being blocked or becoming hidden. The trick is that the add-on or browser cannot detect the drawn image in canvas as a normal image represented by tag <img> of HTML or CSS; therefore, it survives the block. However, free basics service by Facebook has another way of blocking images when free mode is enabled on $3 \mathrm{G}$ or $4 \mathrm{G}$ networks, which are tested in the next section. 

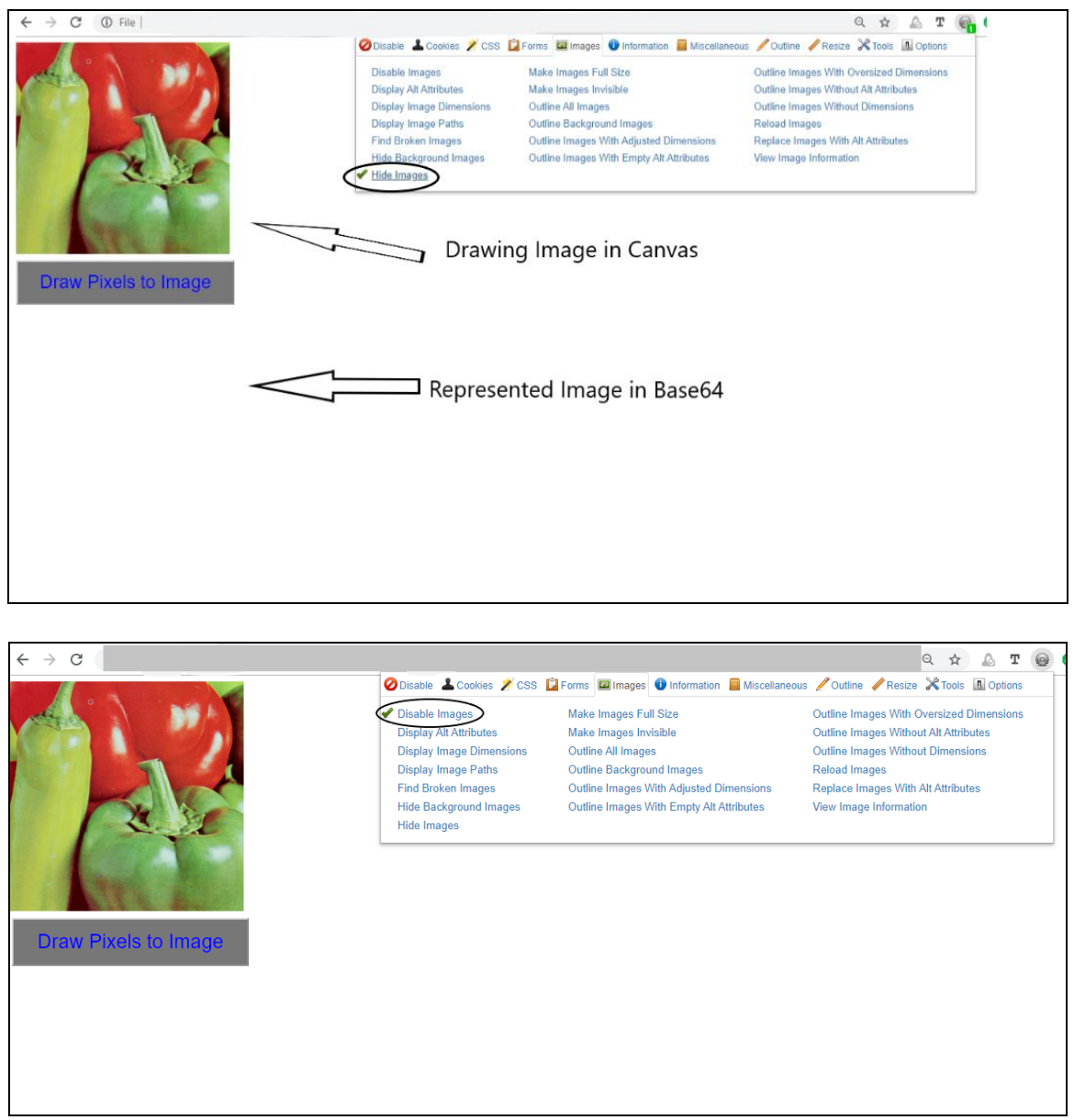

Fig. 6. Blocking and Hiding Images on Browser by Add-on.

\subsection{Test on free basics service by Facebook}

Facebook can be accessed by $3 \mathrm{G}$ or $4 \mathrm{G}$ services for free with no paid subscription to mobile service provider but as text only, without the ability to see photos. Images generated by the algorithm proposed in this paper can be published on Facebook and still be available for users without a paid subscription.

We created a Facebook page [24] and uploaded images classified into two types: images generated with the proposed algorithm and normal images used by users on Facebook and then tested availability of the images. The smartphone was connected to 3G network Asia Cell Company, the service provider in Iraq that supports free basics service from Facebook. The Facebook application on smartphone setup uses a free mode which does not show images, only text. Fig. 7 shows the Facebook page where the free mode is enabled and normal images with .png extension do not show up due to the policy of free mode that supports text only. 


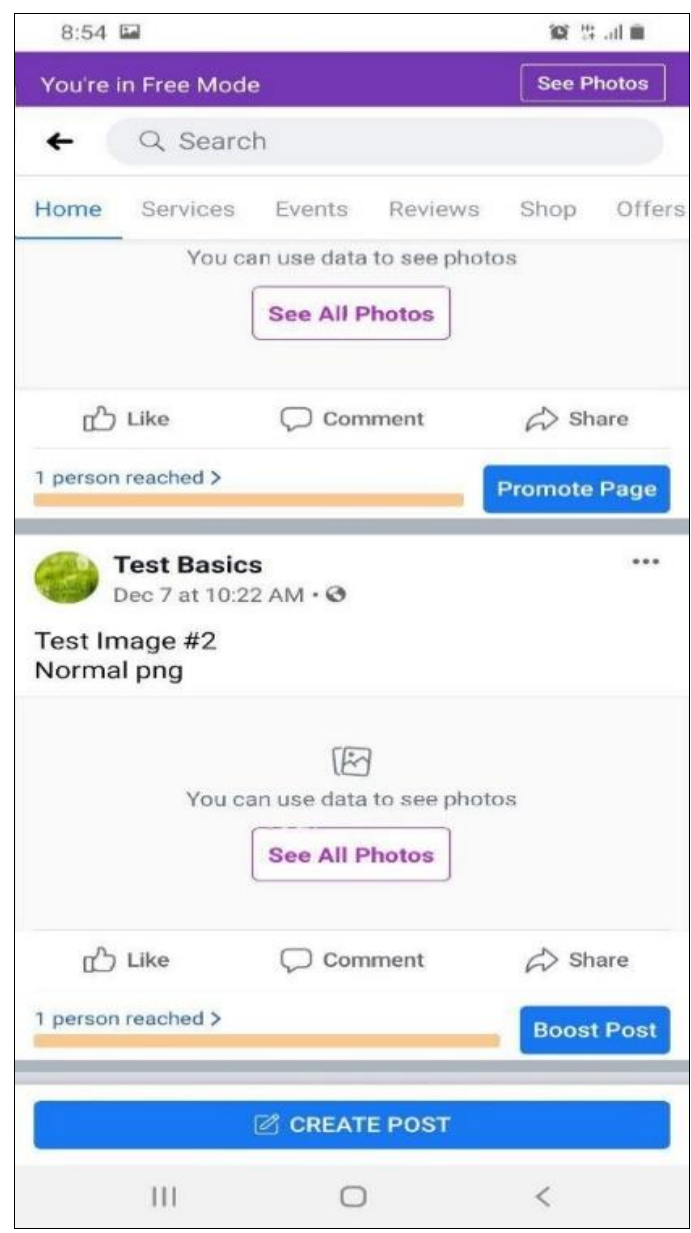

Fig. 7. Free Mode enabled with Free Basics Service

Fig. 8 shows that even though free mode is enabled with Free Basics service, the image produced by the proposed algorithm is still available and can be downloaded as a .png image. The technique we used to produce the image overtakes the free mode procedure by representing the image as a series of alphanumeric representation. This technique treats the image as text and, therefore, can pass the free mode and still be seen by users. 


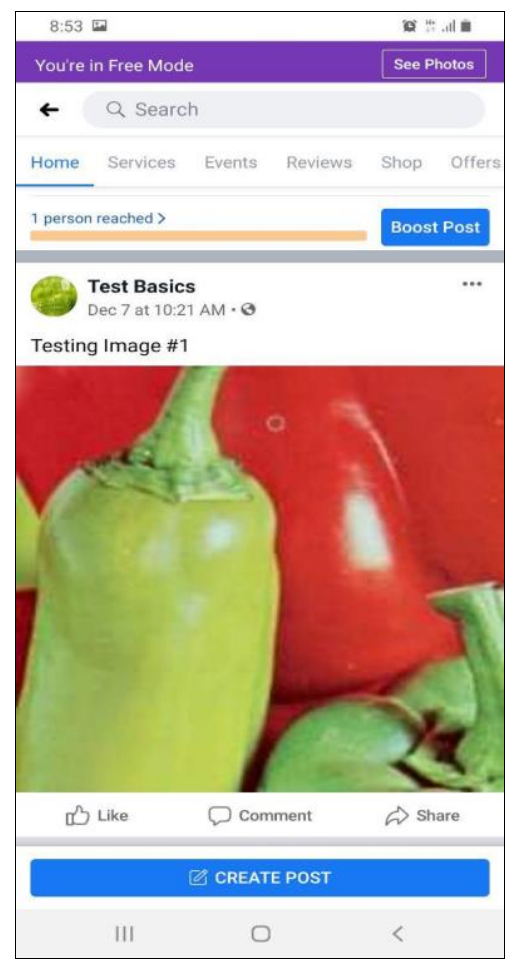

Fig. 8. Image Available with Free Basics and Free Mode

Histogram of image created and posted to Facebook is in Fig. 9 while it is the same as the image converted. This means no changes occurred or affected the original image as posted by the user.

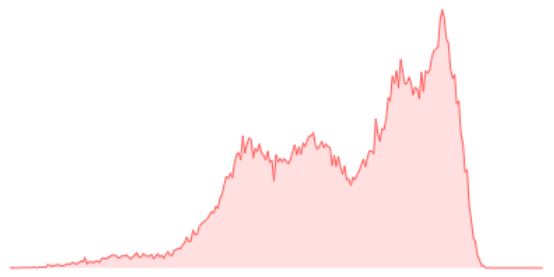

(a) Posted Image histogram

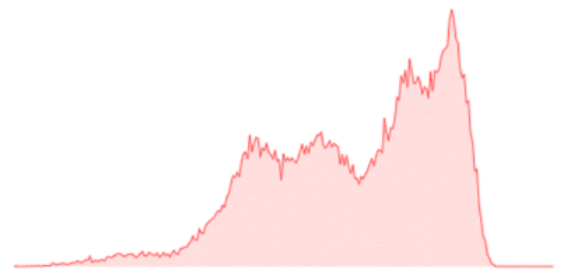

(b) Original Image Histogram

Fig. 9. Histogram of Posted on Facebook Image and Original Image before Conversion

This issue has been reported to Facebook on its White hat page for reporting security issues affecting Facebook functionality. The report screenshot and response from the Facebook team is shown in Fig. 10. The issue was fixed by the Facebook team shortly after reporting the issue. However, the canvas representation of the image can still be possible to show images on free basics service, but Facebook does not allow 
changing the source code of the pages; therefore, we cannot test it at least until after we've written this manuscript.

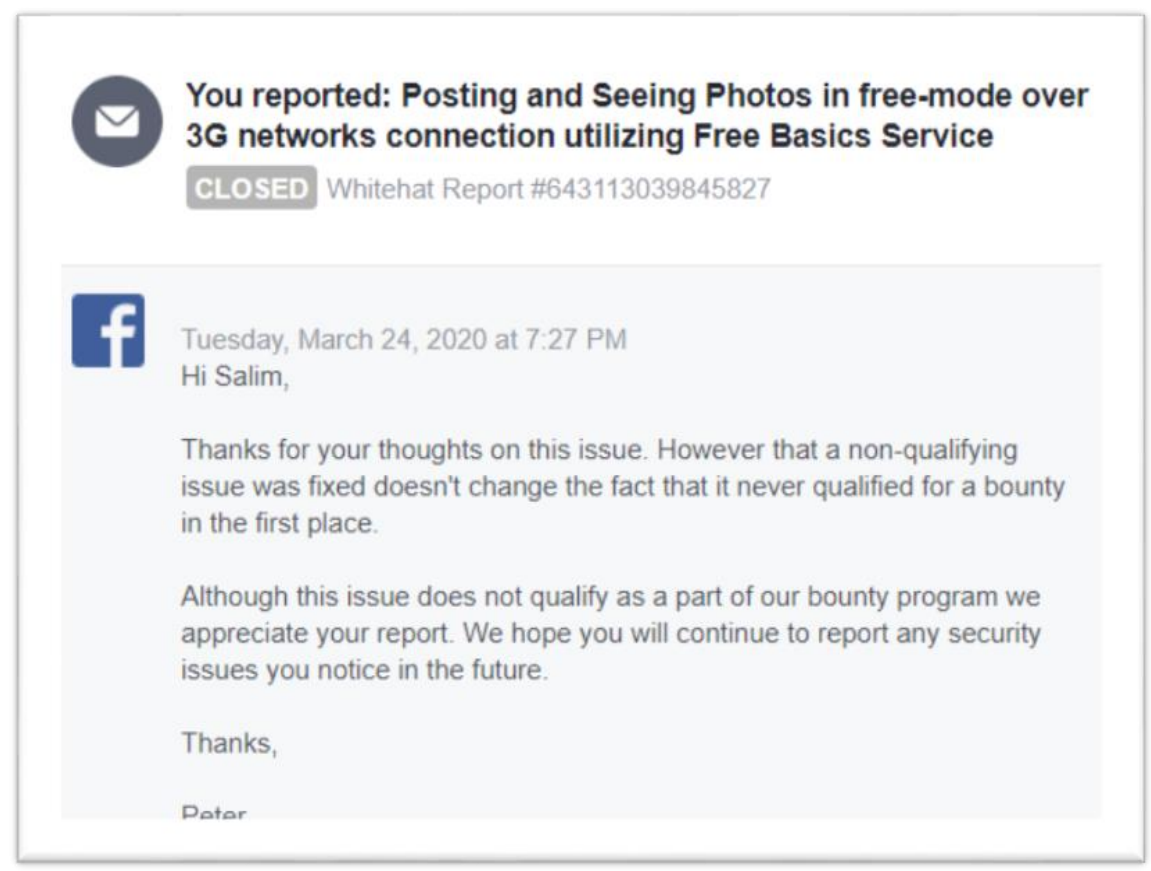

Fig. 10. Report and Response from Facebook Team on the Issue

\section{Conclusion}

The image format conversion presented in this paper could overcome the limitation of showing images in text-only networks. The code in JavaScript transformed the normal image into the pixels' RGB values and positions; then these values were converted into the Base64 image. The applications and network will deal with transferred data as text data, JavaScript and HTML5 codes. This will overcome the limitation of banning images from being shown on applications such as the Facebook app connected over the $3 \mathrm{G}$ network. The image format can be manipulated like any other image such as dimensions and downloadable as .png with a size very close to the size of normal images used on the web. Future work could include applying the premise to videos to check its validity on video service websites. Even though the issues were solved by the Facebook team, still the idea is applicable over text-only networks. A future direction for this study is to decrease the size of the converted image while maintaining the resolution. 


\section{References}

[1] M. Kodad and E. M. Jaara, "Towards Increasing Feedbacks and Diffusion of Information in Social Networks," Int. J. Recent Contrib. from Eng. Sci. IT, vol. 4, no. 1, pp. 11-18, 2016, https://doi.org/10.3991/ijes.v4i1.5477

[2] V. C. Douglas Kunda, Mumbi Chishimba, Mwenge Mulenga, "An Analysis of Security and Performance Concerns in Mobile Web Application Development: Challenges and Open Issues,” Int. J. Recent Contrib. from Eng. Sci. IT, vol. 5, no. 3, pp. 26-40, 2017, https://doi.org/10.3991/ijes.v5i3.7330

[3] C. Facebook, "Our Approach - internet.org," 2020. https://info.internet.org/en/approach/.

[4] S. Singh, I. Qazi, D. Choffnes, and K. P. Gummadi, “An Empirical Analysis of Facebook's Free Basics Program," in ACM SIGMETRICS Performance Evaluation Review, 2017, pp. 37-38, https://doi.org/10.1145/3143314.3078554

[5] M. Yim, R. Gomez, and M. Carter, “Facebook's 'Free Basics' and Implications for Development: IT Identity and Social Capital," in 50th Hawaii International Conference on System Sciences 2017, 2017, pp. 2590-2599, https://doi.org/10.24251/hicss.2017.313

[6] A. Scheerder, A. Deursen, and J. Dijk, "Determinants of Internet skills, uses and outcomes. A systematic review of the second- and third-level digital divide," Telemat. Informatics, vol. 34, no. 8, pp. 1607-1624, 2017, https://doi.org/10.1016/j.tele.2017.07.007

[7] K. P. Sen, Rijurekha \& Pirzada, Hasnain \& Phokeer, Amreesh \& Farooq, Zaid \& Sengupta, Satadal \& Choffnes, David \& Gummadi, "On the Free Bridge Across the Digital Divide: Assessing the Quality of Facebook's Free Basics Service," in IMC - ACM, 2016, pp. 127-133, https://doi.org/10.1145/2987443.2987485

[8] A. Chen, N. Feamster, and E. Calandro, "Exploring the walled garden theory: An empirical framework to assess pricing effects on mobile data usage," Telecomm. Policy, vol. 41, no. 7-8, pp. 587-599, 2017, https://doi.org/10.1016/j.telpol.2017.07.002

[9] T. Saptariani, S. Madenda, and W. S. Ernastuti, "Accelerating Compression Time of the Standard JPEG by Employing the Quantized YCbCr Color Space Algorithm," Int. J. Electr. Comput. Eng., vol. 8, no. 6, pp. 4343-4351, 2018, https://doi.org/10.11591/ijece. v8i6.pp4343-4351

[10] H. T. Wibisono Sukmo Wardhono, Nurizal Dwi Priandani, Mahardeka Tri Ananta, Komang Candra Brata, "End-to-End Privacy Protection for Facebook Mobile Chat based on AES with Multi-Layered MD5," Int. J. Interact. Mob. Technol., vol. 12, no. 1, pp. 160167, 2018. https://doi.org/10.3991/ijim.v12i1.7472

[11] A. V. Rozhgar Dhyab, "Distance Education Features using Facebook," Int. J. Interact. Mob. Technol., vol. 12, no. 6, pp. 19-34, 2018, https://doi.org/10.3991/ijim.v12i6.9621

[12] L. Cilliers and A. Samarthya-Howard, "'Everyone Will Be Connected': Free Basics in Africa to Support ICT4D," in Affordability Issues Surrounding the Use of ICT for Development and Poverty Reduction, 2018, pp. 28-47. https://doi.org/10.4018/978-1-5225-3179$\underline{1 . \operatorname{ch} 002}$

[13] R. Sen et al., "Inside the Walled Garden: Deconstructing Facebook's Free Basics Program," SIGCOMM Comput. Commun. Rev., vol. 47, no. 5, pp. 12-24, 2017, https://doi. org/10.1145/3155055.3155058

[14] M. C. Julianne Romanosky, "Understanding the Use and Impact of the Zero-Rated Free Basics Platform in South Africa," in In Proceedings of the 2018 CHI Conference on Human Factors in Computing Systems (CHI '18), 2018, pp. 1-13, https://doi.org/10.1145/ $\underline{3173574.3173766}$

[15] E. Dimogerontakis, R. Meseguer, L. Navarro, S. Ochoa, and L. Veiga, "Design trade-offs of crowdsourced web access in community networks," in IEEE 21 st International Confer- 
ence on Computer Supported Cooperative Work in Design (CSCWD), 2019, pp. 24-29, https://doi.org/10.1109/cscwd.2017.8066665

[16] M. Zhao, A. Laszka, T. Maillart, and J. Grossklags, "Crowdsourced Security Vulnerability Discovery: Modeling and Organizing Bug-Bounty Programs," 2016, pp. 1-4. https://doi. org/10.5325/jinfopoli.7.2017.0372

[17] J. Ruohonen and L. Allodi, "A Bug Bounty Perspective on the Disclosure of Web Vulnerabilities," in 17th Annual Workshop on the Economics of Information Security, Innsbruck, 2018, pp. 1-14, doi: arXiv:1805.09850v1.

[18] Mark J. Burge and W. Burger, Digital Image Processing: An Algorithmic Introduction Using Java. Springer, 2008.

[19] S. K., "Digital Image Formats," in Digital Image Forensics, S. H. and M. N., Eds. New York, NY: Springer, 2013, pp. 79-121. https://doi.org/10.1007/978-1-4614-0757-7_3

[20] C. Bhabatosh', majumder D. Dutta, and, Digital Image Processing and Analysis, Second. New Delhi: PHI Learning Private Limited, 2011.

[21] E. Weyl, Mobile HTML5: Using the Latest Today. O’Reilly Media, Inc., 2014.

[22] S. Powers, Painting the Web. O'Reilly Media, Inc., 2008.

[23] MDN contributors, "Base64," OnLine, 2020. https://developer.mozilla.org/en-US/docs/ Glossary/Base64.

[24] Salim M. Zaki, "Test Basics," Online, Facebook.com, 2020. http://www.facebook.com/ testbasics/.

\section{$7 \quad$ Author}

Salim M. Zaki is currently a senior lecturer at department of computer science at Dijlah University College, Iraq. His main research interest is related to information security, social networks and mobile networks.

Article submitted 2020-09-27. Resubmitted 2020-12-05. Final acceptance 2020-12-06. Final version published as submitted by the authors. 